\title{
Lifestyle and Comorbidity-Related Risk Factors of Severe and Critical COVID-19 Infection: A Comparative Study Among Survived COVID-19 Patients in Bangladesh
}

\author{
Faroque Md Mohsin (iD ${ }^{1,2}$ \\ Ridwana Nahrin (D) ${ }^{2}$ \\ Tajrin Tahrin Tonmon (D) ${ }^{2}$ \\ Maherun $\mathrm{Nesa}^{3}$ \\ Sharmin Ahmed Tithy (D) \\ Shuvajit Saha ${ }^{4}$ \\ Mahmudul Mannan ${ }^{5}$ \\ Md Shahjalal (D) ${ }^{2}$ \\ Mohammad Omar Faruque ${ }^{6}$ \\ Mohammad Delwer Hossain \\ Hawlader (iD ${ }^{2}$ \\ 'Directorate General of Health Services, \\ Ministry of Health and Family Welfare, \\ Dhaka, Bangladesh; ${ }^{2}$ Department of \\ Public Health, North South University, \\ Dhaka, Bangladesh; ${ }^{3}$ Department of \\ Surgery, Sheikh Hasina National Institute \\ of Burn \& Plastic Surgery, Dhaka, \\ Bangladesh; ${ }^{4}$ Department of Maternal and \\ Child Health, Projahnmo Research \\ Foundation, Dhaka, Bangladesh; \\ ${ }^{5}$ Department of Health Research \\ Methods, Evidence and Impact, McMaster \\ University, Hamilton, Canada; \\ ${ }^{6}$ Department of Botany, University of \\ Chittagong, Chattogram, Bangladesh
}

Correspondence: Mohammad Delwer Hossain Hawlader

Department of Public Health, North

South University, Dhaka, 1229,

Bangladesh

Email mohammad.hawlader@northsouth. edu
Background: Severe COVID-19 infections have already taken more than 4 million lives worldwide. Factors, such as socio-demographics, comorbidities, lifestyles, environment, and so on, have been widely discussed to be associated with increased severity in many countries. The study aimed to determine the risk factors of severe-critical COVID-19 in Bangladesh. Methods: This was a comparative cross-sectional study among various types of COVID-19 patients (both hospitalized and non-hospitalized) confirmed by reverse transcription polymerase chain reaction (RT-PCR). We have selected 1500 COVID-19 positive patients using a convenient sampling technique and analyzed lifestyle and comorbidity-related data using IBM SPSS-23 statistical package software. Chi-square test and multinomial logistic regression were used to determine risk factors of life-threatening COVID-19 infection.

Results: The mean age of the study participants was $43.23( \pm 15.48)$ years. The study identified several lifestyle-related factors and common commodities as risk factors for severe-critical COVID-19. The patient's age was one of the most important predictors, as people $>59$ years were at higher risk $(\mathrm{AOR}=18.223)$. Among other lifestyle factors, active smoking $(\mathrm{AOR}=1.482)$, exposure to secondary smoking ( $\mathrm{AOR}=1.728)$, sleep disturbance $(\mathrm{AOR}=2.208)$ and attachment with $\mathrm{SLT} /$ alcohol/substance abuse $(\mathrm{AOR}=1.804)$ were identified as significant predictors for severe-critical COVID-19. Patients those were overweight/ obese $(\mathrm{AOR}=2.105)$, diabetic $(\mathrm{AOR}=4.286)$, hypertensive $(\mathrm{AOR}=3.363), \mathrm{CKD}$ patients $(\mathrm{AOR}=8.317)$, asthma patients $(\mathrm{AOR}=2.152), \mathrm{CVD}$ patients $(\mathrm{AOR}=7.747)$ were also at higher risk of severe-critical COVID-19 infection.

Conclusion: This study has identified several vital lifestyles and comorbidity-related risk factors of severe-critical COVID-19. People who have these comorbidities should be under high protection, and risky lifestyles of the general population should modify through the proper educational campaign.

Keywords: COVID-19, lifestyle, comorbidities, risk factor, Bangladesh

\section{Introduction}

The virus diseases are highly infectious, and risk depends upon the characteristics of the susceptible host and the virus itself. ${ }^{1}$ There is a rich history of a viral pandemic, and viral diseases have always been a widely discussed public health issue. The emergence of severe acute respiratory syndrome-coronavirus (SARSCoV) during 2002-2003 and novel influenza A (H1N1) in 2009 were two recent 
major viral threats. ${ }^{2}$ However, the emergence of Corona Virus Disease 2019 (COVID-19) is the most serious threat of the 21 st century, which has been announced as a pandemic by the World Health Organization (WHO) on March 11, 2020. ${ }^{3}$ Until September 16, 2021, globally, 226,173,361 cases and 4,655,442 deaths were recorded due to COVID-19. USA, India, and United Kingdom are the top three countries facing the burden of disease, and Bangladesh is one of those 30 countries having a large number of cases of COVID-19. Until September 16, 2021, a total of 1,536,341 cases had been detected, and more than twenty-seven thousand people died due to COVID-19 in Bangladesh. ${ }^{4}$ The number of cases and deaths has been increasing more rapidly than in previous days due to the emergence of a new variant of COVID-19. ${ }^{5}$

Various socio-demographic factors such as age, BMI, gender, occupation, education and residential status would have a significant impact on COVID-19 severity. There are also a number of health-related lifestyle factors such as smoking, unhealthy food habit, alcoholism, substance abuse, physical inactivity, sedentary activity, regular breakfast, water consumption, and so on. Some studies have recently reported the impact of obesity on the severity of COVID-19 infection. ${ }^{6,7}$ The impact of smoking on severe COVID-19 is controversial; however, the Centre for Disease Control and Prevention identified obesity and smoking as a determinant of severe COVID-19 illness. ${ }^{8}$ Several recently conducted studies have observed the association of lifestyle-related factors with morbidity, mortality, and loss of disease-free years of life. ${ }^{9-11}$ According to some previously conducted research, advanced age, asthma, diabetes mellitus, chronic kidney disease (CKD), liver problem, cardiovascular disease, and other immunodeficiency are associated factors of severe COVID-19 infection. ${ }^{12-16}$ The smokers, as well as substance abusers and smokeless tobacco (SLT) takers, would also be immunocompromised due to weakness in the lungs and other relevant organs. ${ }^{17} \mathrm{~A}$ recent study conducted in USA identified higher risk COVID-19 among individuals with of greater sleep problems. ${ }^{18}$ According to Hamer et al, some common lifestyle-related factors such as physical activity, smoking, and obesity increase the odds of severe COVID-19 disease. ${ }^{19}$

In Bangladesh, there is a high prevalence of diabetes, hypertension, chronic kidney disease, and cardiovascular diseases. ${ }^{20}$ With a large number of such immunocompromised people in Bangladesh, there is a higher chance of death due to COVID-19 infection and post-COVID-19 symptoms. A previously conducted study between 2009 and 2012 observed that $23 \%$ of the adult population above 15 years was involved in smoking, and a large portion $(53.5 \%)$ was exposed to secondhand smoking. ${ }^{21,22}$ Therefore, in Bangladesh, there are a large proportion of direct and secondary smokers, which may cause a greater burden of severe infection of COVID-19. ${ }^{23}$ Since the emergence of COVID-19 in Bangladesh, a large number of studies have been conducted focusing on various issues related to COVID-19. However, no study has been published focusing on lifestyle and comorbidity-related risk factors. A study focusing on lifestyle and comorbidities is very much important as the health-related lifestyle is poor in Bangladesh. Therefore, our aim was to determine the lifestyle and comorbidity-related risk factors of severe and critical COVID-19 infections in Bangladesh.

\section{Materials and Methods Study Design}

This study was a hospital-based comparative cross-sectional study where we have selected samples from the hospitals. We have conveniently chosen three hospitals from Dhaka City Corporation for sample selection. We have collected data through face-to-face interviews and telephone conversations with COVID-19 positive patients confirmed by Reverse Transcription Polymerase Chain Reaction (RT-PCR) from November 2020 to April 2021. Patients were categorized based on the severity of COVID-19 symptoms.

We defined the severity of COVID-19 according to the definition of The Bangladesh Society of Medicine. Following their instruction, we have categorized COVID19 patients as (a) Asymptomatic, (b) Mild, (c) Moderate, (d) Severe, and (e) Critical based on the severity of symptom. $^{24}$ The Center for Disease Control and Prevention (CDC) has categorized smokers as people who have smoked 100 or more than 100 cigarettes and are continuing. ${ }^{15}$ People who did not smoke at least 100 cigarettes were ever defined as non-smokers. We have followed these definitions to identify various types of smokers. To shorten the interview period validated questionnaire to measure physical activity, sleep disturbance was not included in the final interview questionnaire. Initially, the questionnaire was developed including a validated questionnaire to measure physical activity level and sleep disturbance. However, during the pretest session, we face difficulties to complete the interview. Therefore, we have simply addressed amount of regular physical activity 
(which they perform at least 5 days a week), daily sedentary hour and the quality of sleep.

\section{Measures}

We have further categorized the COVID-19 cases into two broad categories: (a) Life-threatening COVID-19 consist of severe and critical cases, (b) Non-life threatening COVID19 consists of asymptomatic, mild, and moderate cases. An equal number $(\mathrm{n}=500)$ of samples were recruited from each of the hospitals, which also included an equal number of asymptomatic, mild, moderate, severe, and critical patients (100 patients from each group). Patients were asked regarding their lifestyle and comorbidity history (Diabetes, Hypertension, CKD, Asthma, CVD, COPD) before being infected with COVID-19. Finally, we have included 1500 complete interviews for the analysis.

\section{Statistical Analysis}

We performed data analysis using IBM SPSS version 23 statistical package software. We conducted descriptive statistics to determine the mean, frequency, and percentage to characterize the COVID-19 patients. We conducted a Chisquare test to determine the significantly associated factors with severe and critical COVID-19 infections, and those variables of chi-square analysis were subjected to a multinomial logistic regression analysis to assess the lifestyle and comorbidity-related risk factors of life-threatening COVID-19 disease. We reported the results as adjusted odds ratios (AORs) with 95\% confidence intervals (CIs). A p-value $<0.05$ was considered statistically significant.

\section{Ethical Issue}

This study was approved by the institution's review board (IRB)/ethical review committee (ERC) of North South University (2020/OR-NSU/IRB-No.0802), and each of the steps of this study was conducted in accordance with the Declaration of Helsinki. An informed written consent was obtained from all the participants who attended the face-toface interview. The nature, purpose, and objective of the study were clearly described along with the declaration of confidentiality and anonymity during the virtual interview.

\section{Results}

\section{Descriptive Statistics of Explanatory Variables}

The mean age of the study participants was 43.23 $( \pm 15.48)$, and a large portion of them was of advance aged $45-59$ years $(24.6 \%),>59$ years $(18.0 \%)$. Among the participants, $69.2 \%$ were males, $78.1 \%$ were married, $90.9 \%$ were from urban residences, and $91.2 \%$ were Muslim. Private services (32.1\%), Business (20.3\%), and Housewife (15.3\%) were the three most common occupations reported by the respondents. More than $45 \%$ of respondents had comorbidity, among comorbidities, hypertension (30.4\%), diabetes mellitus (25.2\%), asthma (14.8\%), CVD (5.7\%), COPD (3.4\%), and CKD (2.9\%) were most prevalent. Overall, $26.4 \%$ of patients were smokers before COVID-19 infection, and 32.3\% said they were exposed to secondary smoking. Only $17.2 \%$ did adequate physical activity, the prevalence of sleep disturbance was also high (36.8\%) among the study participants. The habit of having a regular breakfast was seen among $70 \%$ of participants, $55.1 \%$ drunk at least eight glasses of water per day. Moreover, we have pooled all types of smokeless tobacco/substance abuse/alcoholism into one variable, and $38.1 \%$ had at least one of those unhealthy habits (Table 1). This table also describes the scenario of COVID-19 severity under various explanatory variables. Our analysis indicates that the severity of COVID-19 increases with age and BMI. The percentage of severe and critical cases was high in the presence of various comorbidities. Prevalence of life-threatening COVID-19 was also high among smokers, physically less active, or have sleep disturbance.

\section{Association Between Explanatory Variables and Severity of COVID-19}

The association between the explanatory variables and the severity of COVID-19 was determined by the Chi-square test. Among the socio-demographic variables, the lifethreatening COVID-19 infections were significantly associated with the age of the patients, BMI, and Gender $(p<0.05)$. On the other hand, all the considered comorbidities had a statistically significant association with COVID-19 severity. Moreover, among the lifestyle related characteristics, both types of smoking, sleep disturbance, and any types of substance abuse/SLT/alcohol had a statistically significant association with life-threatening COVID-19 $(p<0.05)$ (Table 2).

\section{Risk Factors for Severe and Critical COVID-19 Infections}

The patient's age was one of the most important predictors, as older peoples were at higher risk $(\mathrm{AOR}=18.223$, 
Table I Descriptive Statistics of Explanatory Variables with Types of COVID-I9 Patients

\begin{tabular}{|c|c|c|c|c|c|c|}
\hline Variables & Frequency (\%) & Asymptomatic (\%) & Mild (\%) & Moderate (\%) & Severe (\%) & Critical (\%) \\
\hline $\begin{array}{l}\text { Age } \\
\begin{array}{l}<19 \\
19-34 \\
35-44 \\
45-59 \\
>59\end{array}\end{array}$ & $\begin{array}{c}26(1.7) \\
509(33.9) \\
326(21.7) \\
369(24.6) \\
270(18.0)\end{array}$ & $\begin{array}{c}16(61.5) \\
156(30.6) \\
86(26.4) \\
27(7.3) \\
15(5.5)\end{array}$ & $\begin{array}{c}5(19.2) \\
228(44.8) \\
45(13.8) \\
19(5.1) \\
3(1.1)\end{array}$ & $\begin{array}{l}4(15.4) \\
89(17.5) \\
99(30.4) \\
87(23.6) \\
21(7.8)\end{array}$ & $\begin{array}{c}0(0.0) \\
23(4.5) \\
64(19.6) \\
128(34.7) \\
85(31.5)\end{array}$ & $\begin{array}{c}1(3.8) \\
13(2.6) \\
32(9.8) \\
108(29.3) \\
146(54.1)\end{array}$ \\
\hline $\begin{array}{l}\text { BMI } \\
\text { Underweight } \\
\text { Normal } \\
\text { Overweight } \\
\text { Obesity }\end{array}$ & $\begin{array}{c}20(1.3) \\
719(47.9) \\
607(40.5) \\
152(10.1)\end{array}$ & $\begin{array}{l}13(65.0) \\
160(22.3) \\
102(16.8) \\
25(16.4)\end{array}$ & $\begin{array}{c}2(10.0) \\
170(23.6) \\
106(17.5) \\
21(13.8)\end{array}$ & $\begin{array}{c}3(15.0) \\
160(22.3) \\
105(17.3) \\
31(20.4)\end{array}$ & $\begin{array}{c}\text { I (5.0) } \\
138(19.2) \\
130(21.4) \\
31(20.4)\end{array}$ & $\begin{array}{l}\text { I (5.0) } \\
91(12.7) \\
164(27.0) \\
44(28.9)\end{array}$ \\
\hline $\begin{array}{l}\text { Gender } \\
\text { Female } \\
\text { Male }\end{array}$ & $\begin{array}{c}462(30.8) \\
1038(69.2)\end{array}$ & $\begin{array}{l}106(22.9) \\
194(18.7)\end{array}$ & $\begin{array}{c}99(21.4) \\
201(19.4)\end{array}$ & $\begin{array}{c}94(20.3) \\
206(19.8)\end{array}$ & $\begin{array}{c}94(20.3) \\
206(19.8)\end{array}$ & $\begin{array}{c}69(14.9) \\
231(22.3)\end{array}$ \\
\hline $\begin{array}{l}\text { Occupation } \\
\text { Business } \\
\text { Doctor } \\
\text { Other health } \\
\text { Service (govt) } \\
\text { Service (private) } \\
\text { Housewife } \\
\text { Student } \\
\text { Worker } \\
\text { Unemployed } \\
\text { Retired } \\
\text { Others }\end{array}$ & $\begin{array}{c}305(20.3) \\
21(1.4) \\
32(2.1) \\
45(3.0) \\
481(32.1) \\
230(15.3) \\
86(5.7) \\
109(7.3) \\
102(6.8) \\
62(4.1) \\
27(1.8)\end{array}$ & $\begin{array}{c}51(16.7) \\
5(23.8) \\
18(56.3) \\
6(13.3) \\
92(19.1) \\
35(15.2) \\
29(33.7) \\
29(26.6) \\
22(21.6) \\
0(0.0) \\
13(48.1)\end{array}$ & $\begin{array}{c}54(17.7) \\
6(28.6) \\
7(21.9) \\
11(24.4) \\
82(17.0) \\
44(19.1) \\
44(51.2) \\
40(36.7) \\
11(10.8) \\
0(0.0) \\
1(3.7)\end{array}$ & $\begin{array}{c}67(22.0) \\
6(28.6) \\
3(9.4) \\
17(37.8) \\
107(22.2) \\
47(20.4) \\
8(9.3) \\
26(23.9) \\
11(10.8) \\
1(1.6) \\
7(25.9)\end{array}$ & $\begin{array}{c}60(19.7) \\
0(0.0) \\
2(6.3) \\
8(17.8) \\
106(22.0) \\
56(24.3) \\
2(2.3) \\
8(7.3) \\
42(41.2) \\
14(22.6) \\
2(7.4)\end{array}$ & $\begin{array}{c}73(23.9) \\
4(19.0) \\
2(6.3) \\
3(6.7) \\
94(19.5) \\
48(20.9) \\
3(3.5) \\
6(5.5) \\
16(15.7) \\
47(75.8) \\
4(14.8)\end{array}$ \\
\hline $\begin{array}{l}\text { Marital status } \\
\text { Unmarried } \\
\text { Married } \\
\text { Divorced/separated }\end{array}$ & $\begin{array}{l}237(15.8) \\
1172(78.1) \\
91(6.1)\end{array}$ & $\begin{array}{c}88(37.1) \\
210(18.1) \\
2(2.2)\end{array}$ & $\begin{array}{c}96(40.5) \\
204(17.5) \\
0(0.0)\end{array}$ & $\begin{array}{c}42(17.7) \\
249(21.3) \\
9(9.9)\end{array}$ & $\begin{array}{c}7(3.0) \\
270(23.1) \\
23(25.3)\end{array}$ & $\begin{array}{c}4(1.7) \\
239(20.4) \\
57(62.6)\end{array}$ \\
\hline $\begin{array}{c}\text { Residence } \\
\text { Urban } \\
\text { Rural }\end{array}$ & $\begin{array}{c}1363(90.9) \\
137(9.1)\end{array}$ & $\begin{array}{c}279(20.5) \\
21(15.3)\end{array}$ & $\begin{array}{c}263(19.3) \\
37(27.0)\end{array}$ & $\begin{array}{c}269(19.7) \\
31(22.6)\end{array}$ & $\begin{array}{c}275(20.2) \\
31(22.6)\end{array}$ & $\begin{array}{c}277(20.3) \\
25(18.2)\end{array}$ \\
\hline $\begin{array}{l}\text { Education } \\
\text { Uneducated } \\
\text { Primary } \\
\text { Secondary } \\
\text { College/diploma } \\
\text { Graduate/higher }\end{array}$ & $\begin{array}{c}58(3.9) \\
128(8.5) \\
324(21.6) \\
493(32.9) \\
497(33.1)\end{array}$ & $\begin{array}{l}16(27.6) \\
35(27.3) \\
68(21.0) \\
115(23.3) \\
66(13.3)\end{array}$ & $\begin{array}{l}15(25.9) \\
24(18.8) \\
78(24.1) \\
84(17.0) \\
99(19.9)\end{array}$ & $\begin{array}{l}15(25.9) \\
26(20.3) \\
75(23.1) \\
112(22.7) \\
72(14.5)\end{array}$ & $\begin{array}{l}10(17.2) \\
22(17.2) \\
57(17.6) \\
75(15.2) \\
136(27.4)\end{array}$ & $\begin{array}{c}2(3.4) \\
21(16.4) \\
46(14.2) \\
107(21.7) \\
124(24.9)\end{array}$ \\
\hline $\begin{array}{l}\text { Religion } \\
\text { Muslim } \\
\text { Others }\end{array}$ & $\begin{array}{c}1368(91.2) \\
132(8.8)\end{array}$ & $\begin{array}{c}267(19.5) \\
33(25.0)\end{array}$ & $\begin{array}{c}268(19.6) \\
25(18.9)\end{array}$ & $\begin{array}{c}283(20.7) \\
32(24.2)\end{array}$ & $\begin{array}{c}275(20.1) \\
17(12.9)\end{array}$ & $\begin{array}{c}275(20.1) \\
25(18.9)\end{array}$ \\
\hline $\begin{array}{l}\text { Comorbidity } \\
\text { No } \\
\text { Yes }\end{array}$ & $\begin{array}{l}682(45.5) \\
818(54.5)\end{array}$ & $\begin{array}{c}257(37.7) \\
43(5.3)\end{array}$ & $\begin{array}{c}239(35.0) \\
61(7.5)\end{array}$ & $\begin{array}{l}119(17.4) \\
181(22.1)\end{array}$ & $\begin{array}{c}32(4.7) \\
268(32.8)\end{array}$ & $\begin{array}{c}35(5.1) \\
265(32.4)\end{array}$ \\
\hline $\begin{array}{l}\text { Diabetes } \\
\text { No } \\
\text { Yes }\end{array}$ & $\begin{array}{l}\text { II } 22(74.8) \\
378(25.2)\end{array}$ & $\begin{array}{c}289(25.8) \\
\text { II (2.9) }\end{array}$ & $\begin{array}{l}292(26.0) \\
143(37.8)\end{array}$ & $\begin{array}{c}249(22.2) \\
8(2.1)\end{array}$ & $\begin{array}{c}135(12.0) \\
5 \mid(13.5)\end{array}$ & $\begin{array}{l}157(14.0) \\
143(37.8)\end{array}$ \\
\hline
\end{tabular}

(Continued) 
Table I (Continued).

\begin{tabular}{|c|c|c|c|c|c|c|}
\hline Variables & Frequency (\%) & Asymptomatic (\%) & Mild (\%) & Moderate (\%) & Severe (\%) & Critical (\%) \\
\hline \multicolumn{7}{|l|}{ Hypertension } \\
\hline No & $1044(69.6)$ & $287(27.5)$ & $280(26.8)$ & $229(21.9)$ & $119(11.4)$ & $129(12.4)$ \\
\hline Yes & $456(30.4)$ & $13(2.9)$ & $20(4.4)$ & 71 (15.6) & 181 (39.7) & $|7|(37.5)$ \\
\hline \multicolumn{7}{|l|}{ CKD } \\
\hline No & 1457 (97.1) & $299(20.5)$ & $298(20.5)$ & $300(20.6)$ & $289(19.8)$ & $271(18.6)$ \\
\hline Yes & $43(2.9)$ & I (2.3) & $2(4.7)$ & $0(0.0)$ & II (25.6) & $29(67.4)$ \\
\hline \multicolumn{7}{|l|}{ Asthma } \\
\hline No & $1278(85.2)$ & $279(21.8)$ & $279(21.8)$ & $232(18.2)$ & $221(17.3)$ & $267(20.9)$ \\
\hline Yes & $222(14.8)$ & $21(9.5)$ & $21(9.5)$ & $68(30.6)$ & $79(35.6)$ & $33(14.9)$ \\
\hline \multicolumn{7}{|l|}{ CVD } \\
\hline No & $1415(94.3)$ & $300(21.2)$ & $300(21.2)$ & $294(20.8)$ & $26 \mid(18.4)$ & $260(18.4)$ \\
\hline Yes & $85(5.7)$ & $0(0.0)$ & $0(0.0)$ & $6(7.1)$ & $39(45.9)$ & $40(47.1)$ \\
\hline \multicolumn{7}{|l|}{ COPD } \\
\hline No & 1449 (96.6) & $300(20.7)$ & $300(20.7)$ & $290(20.0)$ & $277(19.1)$ & $282(19.5)$ \\
\hline Yes & $51(3.4)$ & $0(0.0)$ & $0(0.0)$ & $10(19.6)$ & $23(45.1)$ & $18(35.3)$ \\
\hline \multicolumn{7}{|l|}{ Smoking type } \\
\hline Current & $396(26.4)$ & $26(6.6)$ & 75 (18.9) & $97(24.5)$ & $108(27.3)$ & $90(22.7)$ \\
\hline Former & $99(6.6)$ & $20(20.2)$ & $27(27.3)$ & $13(13.1)$ & $29(29.3)$ & $10(10.1)$ \\
\hline Never & $933(62.2)$ & $240(25.7)$ & $184(19.7)$ & $|7|(18.3)$ & $153(16.4)$ & $185(19.8)$ \\
\hline Occasional & $72(4.8)$ & $14(19.4)$ & $14(19.4)$ & $19(26.4)$ & $10(13.9)$ & $15(20.8)$ \\
\hline \multicolumn{7}{|c|}{ Secondary smoking } \\
\hline Do not know & $332(22.1)$ & $76(22.9)$ & $68(20.5)$ & $63(19.0)$ & $68(20.5)$ & $57(17.2)$ \\
\hline No & $683(45.5)$ & $146(21.4)$ & $119(17.4)$ & $167(24.5)$ & $121(17.7)$ & $130(19.0)$ \\
\hline Yes & $485(32.3)$ & $78(16.1)$ & $113(23.3)$ & $70(14.4)$ & III (22.9) & $113(23.3)$ \\
\hline \multicolumn{7}{|l|}{ Physical activity } \\
\hline No & $544(36.3)$ & $106(19.5)$ & $109(20.0)$ & $105(19.3)$ & $105(19.3)$ & $119(21.9)$ \\
\hline$<30$ minutes & $698(46.5)$ & $140(20.1)$ & $133(19.1)$ & $135(19.3)$ & $146(20.9)$ & $144(20.6)$ \\
\hline$>30$ minutes & $258(17.2)$ & $54(20.9)$ & $58(22.5)$ & $60(23.3)$ & $49(19.0)$ & $37(14.3)$ \\
\hline \multicolumn{7}{|l|}{ Sleep disturbance } \\
\hline No & $948(63.2)$ & $203(21.4)$ & $207(21.8)$ & $211(22.3)$ & $157(16.6)$ & $170(17.9)$ \\
\hline Yes & $552(36.8)$ & $97(17.6)$ & $93(16.8)$ & $89(16.1)$ & $143(25.9)$ & $130(23.6)$ \\
\hline \multicolumn{7}{|l|}{ Sedentary hour } \\
\hline$<2$ hours & $474(31.6)$ & $90(19.0)$ & 98 (20.7) & $95(20.0)$ & $94(19.8)$ & $97(20.5)$ \\
\hline $2-4$ hours & $366(24.4)$ & 71 (19.4) & $73(19.9)$ & 73 (19.9) & $78(21.3)$ & 71 (19.4) \\
\hline$>4$ hours & $660(44.0)$ & $139(21.1)$ & $129(19.5)$ & $132(20.0)$ & $128(19.4)$ & $132(20.0)$ \\
\hline \multicolumn{7}{|l|}{ Alcohol/SLT/others } \\
\hline No & $928(61.9)$ & $211(22.7)$ & $217(23.4)$ & $198(21.3)$ & $168(18.1)$ & $134(14.4)$ \\
\hline Yes & $572(38.1)$ & 89 (15.6) & $83(14.5)$ & $102(17.8)$ & $132(23.1)$ & $166(29.0)$ \\
\hline \multicolumn{7}{|l|}{ Regular breakfast } \\
\hline No & $450(30.0)$ & 89 (19.8) & $89(19.8)$ & $94(20.9)$ & $90(20.0)$ & $88(19.6)$ \\
\hline Yes & $1050(70.0)$ & $211(20.1)$ & $211(20.1)$ & $206(19.6)$ & $210(20.0)$ & $212(20.2)$ \\
\hline \multicolumn{7}{|l|}{ Water drinking } \\
\hline No & $674(44.9)$ & $124(18.4)$ & 131 (19.4) & $136(20.2)$ & $130(19.3)$ & 153 (22.7) \\
\hline Yes & $826(55.1)$ & $176(21.3)$ & $169(20.5)$ & $164(19.9)$ & $170(20.6)$ & 147 (I7.8) \\
\hline
\end{tabular}


Table 2 Association Between Severity of COVID-19 and Selected Explanatory Variables

\begin{tabular}{|c|c|c|c|c|}
\hline Variables & Category & Asymptomatic, Mild and Moderate (\%) & Severe and Critical (\%) & $P$ value \\
\hline Age & $\begin{array}{l}<35 \text { years } \\
35-59 \text { years } \\
>59 \text { years }\end{array}$ & $\begin{array}{c}498(93.1) \\
363(52.2) \\
39(14.4)\end{array}$ & $\begin{array}{c}37(6.9) \\
332(47.8) \\
231(85.6)\end{array}$ & $<0.00 I^{*}$ \\
\hline BMI & $\begin{array}{l}\text { Low/normal weight } \\
\text { Overweight/obese }\end{array}$ & $\begin{array}{l}508(68.7) \\
390(51.4)\end{array}$ & $\begin{array}{l}231(31.3) \\
369(48.6)\end{array}$ & $<0.00 I^{*}$ \\
\hline Gender & $\begin{array}{l}\text { Female } \\
\text { Male }\end{array}$ & $\begin{array}{l}299(64.7) \\
601(57.9)\end{array}$ & $\begin{array}{l}163(35.3) \\
437(42.1)\end{array}$ & $0.013^{*}$ \\
\hline Residence & $\begin{array}{l}\text { Rural } \\
\text { Urban }\end{array}$ & $\begin{array}{l}89(65.0) \\
811(59.5)\end{array}$ & $\begin{array}{c}48(35.0) \\
552(40.5)\end{array}$ & 0.213 \\
\hline Comorbidity & $\begin{array}{l}\text { No } \\
\text { Yes }\end{array}$ & $\begin{array}{l}615(90.2) \\
285(34.8)\end{array}$ & $\begin{array}{c}67(9.8) \\
533(65.2)\end{array}$ & $<0.00 I^{*}$ \\
\hline Diabetes & $\begin{array}{l}\text { No } \\
\text { Yes }\end{array}$ & $\begin{array}{c}830(74.0) \\
70(18.5)\end{array}$ & $\begin{array}{l}292(26.0) \\
308(81.5)\end{array}$ & $<0.00 I^{*}$ \\
\hline Hypertension & $\begin{array}{l}\text { No } \\
\text { Yes }\end{array}$ & $\begin{array}{l}796(76.2) \\
104(22.8)\end{array}$ & $\begin{array}{l}248(23.8) \\
352(77.2)\end{array}$ & $<0.00 I^{*}$ \\
\hline CKD & $\begin{array}{l}\text { No } \\
\text { Yes }\end{array}$ & $\begin{array}{c}897(61.6) \\
3(7.0)\end{array}$ & $\begin{array}{l}560(38.4) \\
40(93.0)\end{array}$ & $<0.00 I^{*}$ \\
\hline Asthma & $\begin{array}{l}\text { No } \\
\text { Yes }\end{array}$ & $\begin{array}{l}790(61.8) \\
110(49.5)\end{array}$ & $\begin{array}{l}488(38.2) \\
112(50.5)\end{array}$ & $0.00 I^{*}$ \\
\hline CVD & $\begin{array}{l}\text { No } \\
\text { Yes }\end{array}$ & $\begin{array}{c}894(63.2) \\
6(7.1)\end{array}$ & $\begin{array}{l}521(36.8) \\
79(92.9)\end{array}$ & $<0.00 I^{*}$ \\
\hline COPD & $\begin{array}{l}\text { No } \\
\text { Yes }\end{array}$ & $\begin{array}{c}890(61.4) \\
10(19.6)\end{array}$ & $\begin{array}{c}559(38.6) \\
41(80.0)\end{array}$ & $<0.00 I^{*}$ \\
\hline Smoking type & $\begin{array}{l}\text { Current } \\
\text { Former } \\
\text { Never } \\
\text { Occasional }\end{array}$ & $\begin{array}{c}198(50.0) \\
60(60.6) \\
595(63.8) \\
47(65.3)\end{array}$ & $\begin{array}{c}198(50.0) \\
39(39.4) \\
338(36.2) \\
25(34.7)\end{array}$ & $<0.001 *$ \\
\hline Secondary smoking & $\begin{array}{l}\text { Do not know } \\
\text { No } \\
\text { Yes }\end{array}$ & $\begin{array}{l}207(62.3) \\
432(63.3) \\
261(53.8)\end{array}$ & $\begin{array}{l}125(37.7) \\
25 \mid(36.7) \\
224(46.2)\end{array}$ & $0.003^{*}$ \\
\hline Physical activity & $\begin{array}{l}\text { No } \\
<30 \text { minutes } \\
>30 \text { minutes }\end{array}$ & $\begin{array}{l}320(58.8) \\
408(58.5) \\
172(66.7)\end{array}$ & $\begin{array}{c}224(4 I .2) \\
290(4 I .5) \\
86(33.3)\end{array}$ & 0.055 \\
\hline Sleep disturbance & $\begin{array}{l}\text { No } \\
\text { Yes }\end{array}$ & $\begin{array}{l}621(65.5) \\
279(50.5)\end{array}$ & $\begin{array}{l}327(34.5) \\
273(49.5)\end{array}$ & $<0.00 I^{*}$ \\
\hline Sedentary hour & $\begin{array}{l}<2 \text { hours } \\
2-4 \text { hours } \\
>4 \text { hours }\end{array}$ & $\begin{array}{l}283(59.7) \\
217(59.3) \\
400(60.6)\end{array}$ & $\begin{array}{l}191(40.3) \\
149(40.7) \\
260(39.4)\end{array}$ & 0.907 \\
\hline Alcohol/SLT/others & $\begin{array}{l}\text { No } \\
\text { Yes }\end{array}$ & $\begin{array}{l}626(67.5) \\
274(47.9)\end{array}$ & $\begin{array}{l}302(32.5) \\
298(52.1)\end{array}$ & $<0.00 I^{*}$ \\
\hline Regular breakfast & $\begin{array}{l}\text { No } \\
\text { Yes }\end{array}$ & $\begin{array}{l}272(60.4) \\
628(59.8)\end{array}$ & $\begin{array}{l}178(39.6) \\
422(40.2)\end{array}$ & 0.818 \\
\hline Water drinking & $\begin{array}{l}\text { No } \\
\text { Yes }\end{array}$ & $\begin{array}{l}391(58.0) \\
509(61.6)\end{array}$ & $\begin{array}{l}283(42.0) \\
317(38.4)\end{array}$ & 0.086 \\
\hline
\end{tabular}

Note: ${ }^{*}<0.05$ indicates statistically significant association. 
95\% CI: 10.21-32.53) of severe-critical COVID-19. People those were continuing smoking before COVID-19 had more than $40 \%$ increased risk of severe-critical infection $(\mathrm{AOR}=1.482,95 \% \mathrm{CI}: 1.002-2.194)$. Moreover, exposure to secondary smoking $(\mathrm{AOR}=1.728,95 \% \mathrm{CI}$ : 1.196-2.494) was also a risk factor for life-threatening disease conditions. Participants those fells sleep disturbance had 2.2 times more risk of developing severe infection $(\mathrm{AOR}=2.208,95 \% \mathrm{CI}: 1.601-3.044)$, and attachment with SLT/alcohol/substance abuse $(\mathrm{AOR}=1.804,95 \% \mathrm{CI}$ : 1.319-2.466) is another important risk factor. Patients who were overweight and obese had a higher risk of developing a life-threatening infection than normal-weight individuals ( $\mathrm{AOR}=2.105$, 95\% CI: 1.513-2.927). Various types of comorbidities such as diabetes mellitus (AOR=4.286, 95\% CI: 2.85-6.447), hypertension $(\mathrm{AOR}=3.363,95 \%$ CI: 2.24-5.05), chronic kidney disease (AOR=8.317, 95\% CI: $1.925-35.927)$, asthma $(\mathrm{AOR}=2.152$, 95\% CI: 1.335-3.468), cardiovascular disease $(\mathrm{AOR}=7.747,95 \%$ CI: 2.977-20.16) increase the risk of severe-critical COVID-19 infection (Table 3).

\section{Discussion}

After the initiation of COVID-19 pandemic, a large number of studies have been conducted among the Bangladeshi population. However, most of these were limited to mental health aspect, symptomatology, and impact on health system. A few studies were conducted among COVID-19 cases, and our study is one of them. We have successfully revealed several important risk factors for severe and critical COVID-19 infections in Bangladesh. Here, the number of COVID-19 patients has been increasing, and managing severe and critical cases is a big challenge for the existing health system. Advanced age was identified as an important risk factor of life-threatening COVID-19 as patients who were age 60 years or higher have 18 times more risk of developing severe-critical symptoms of COVID-19 compared to young adults aged less than 35 years $(p<0.001)$. According to CDC, the risk of severe illness due to COVID-19 increases with age. ${ }^{8}$ A study conducted in the UK concludes advanced age as a risk factor for severe symptoms and mortality. ${ }^{25}$ However, some studies also observed a high percentage of young and older adults also infected with life-threatening infections. $^{26-28}$ Being overweight or obese also doubled the risk of severe infection $(\mathrm{AOR}=2.105,95 \% \mathrm{CI}$ : 1.513 2.927). Data from recent studies also suggest that obesity increases the risk for progression from severe to critical
COVID-19 diseases such as cytokine storm, ARDS, and coagulopathy. ${ }^{29}$ Goa et al also identified obesity as a risk factor for advanced severity during COVID-19 infection. ${ }^{30}$

Adult people are earning members of the family; therefore, usually, they require going outside more and need to mix up with people from various locations. Moreover, 35 years or above is a cut-off point for non-communicable diseases development; ${ }^{31}$ therefore, people aged above 35 years are at increased risk of severe COVID-19. ${ }^{32}$ The analysis also shows that various types of common comorbidities increase the risk of severe-critical COVID-19 infections ( $\mathrm{p}<0.001$ ). According to Sanyaolu et al, patients with preexisting comorbidities suffer a severe prognosis of COVID-19. They suggested taking advanced necessary precautions to avoid SARS CoV-2 infection. ${ }^{33}$ Among comorbidities, hypertension was found to be most prevalent in our study, increasing the risk of severe-critical COVID-19 symptoms. A systematic review also found hypertension and obesity to be the most pervasive preexisting comorbidities among COVID-19 patients, and hypertension caused a three-time increase in the risk of severe infection. ${ }^{34}$

Diabetes is the second most common comorbidity reported, and according to our analysis, it causes 4.23 times increase the risk of life-threatening COVID-19 infections. A similar scenario was observed in previously conducted studies, where diabetes was said to increase fatality due to COVID-19. ${ }^{35}$ Various types of chronic lung diseases have an impact on COVID-19 and can make people severely ill. Diseases such as asthma (moderate to severe), chronic obstructive pulmonary disease (COPD), pulmonary fibrosis, and pulmonary hypertension increase the risk of severe infection. ${ }^{8}$ Our analysis also revealed that asthma patients were a high-risk group for severe-critical COVID-19 infections. Patients with asthma problems had 2.15 times higher risk of life-threatening COVID-19 infection $(\mathrm{p}<0.01)$. Though the percentage of CVD and CKD was relatively low in our study, they were also identified as a risk factor for severe and critical COVID-19 infections. It has also been widely discussed that the presence of cardiovascular disease in patients with COVID-19 increases the risk of severe infection as well as mortality. ${ }^{36}$ A study conducted in January 2021 identified chronic kidney disease as a risk factor for severe coronavirus disease. ${ }^{37}$ Moreover, both active and passive smokings are risk factors for advanced COVID-19 symptoms. Regular smokers are at a $48.2 \%$ increased risk of facing severe and critical conditions than the never smokers $(\mathrm{p}<0.001)$, which complies with the 
Table 3 Multiple Adjusted Logistic Regression Analysis to Determine Risk Factors of Severe and Critical COVID-I9 Infections

\begin{tabular}{|c|c|c|c|c|c|}
\hline Variables & Category & Coefficient (B) & AOR & $\mathbf{L C I}$ & UCI \\
\hline Age & $\begin{array}{l}<35 \text { years } \\
35-59 \text { years } \\
>59 \text { years }\end{array}$ & $\begin{array}{l}\text { Reference } \\
1.772 \\
2.903\end{array}$ & $\begin{array}{l}5.881 \\
18.223\end{array}$ & $\begin{array}{l}3.832 \\
10.206\end{array}$ & $\begin{array}{l}9.026 \\
32.539\end{array}$ \\
\hline BMI & $\begin{array}{l}\text { Low/normal weight } \\
\text { Overweight/obese }\end{array}$ & $\begin{array}{l}\text { Reference } \\
0.744\end{array}$ & 2.105 & 1.513 & 2.927 \\
\hline Gender & $\begin{array}{l}\text { Male } \\
\text { Female }\end{array}$ & $\begin{array}{l}\text { Reference } \\
0.131\end{array}$ & 1.14 & 0.759 & 1.713 \\
\hline Comorbidity & $\begin{array}{l}\text { No } \\
\text { Yes }\end{array}$ & $\begin{array}{l}\text { Reference } \\
0.459\end{array}$ & 1.582 & 0.953 & 2.627 \\
\hline Diabetes & $\begin{array}{l}\text { No } \\
\text { Yes }\end{array}$ & $\begin{array}{l}\text { Reference } \\
\text { I.455 }\end{array}$ & 4.286 & 2.85 & 6.447 \\
\hline Hypertension & $\begin{array}{l}\text { No } \\
\text { Yes }\end{array}$ & $\begin{array}{l}\text { Reference } \\
1.213\end{array}$ & 3.363 & 2.24 & 5.05 \\
\hline CKD & $\begin{array}{l}\text { No } \\
\text { Yes }\end{array}$ & $\begin{array}{l}\text { Reference } \\
2.118\end{array}$ & 8.317 & 1.925 & 35.927 \\
\hline Asthma & $\begin{array}{l}\text { No } \\
\text { Yes }\end{array}$ & $\begin{array}{l}\text { Reference } \\
0.766\end{array}$ & 2.152 & 1.335 & 3.468 \\
\hline CVD & $\begin{array}{l}\text { No } \\
\text { Yes }\end{array}$ & $\begin{array}{l}\text { Reference } \\
2.047\end{array}$ & 7.747 & 2.977 & 20.161 \\
\hline COPD & $\begin{array}{l}\text { No } \\
\text { Yes }\end{array}$ & $\begin{array}{l}\text { Reference } \\
0.315\end{array}$ & $1.37 \mid$ & 0.605 & 3.105 \\
\hline Smoking type & $\begin{array}{l}\text { Current } \\
\text { Former } \\
\text { Occasional } \\
\text { Never }\end{array}$ & $\begin{array}{l}0.394 \\
-0.745 \\
-0.306 \\
\text { Reference }\end{array}$ & $\begin{array}{l}1.482 \\
0.475 \\
0.736\end{array}$ & $\begin{array}{l}1.002 \\
0.226 \\
0.344\end{array}$ & $\begin{array}{l}2.194 \\
0.998 \\
1.573\end{array}$ \\
\hline Secondary smoking & $\begin{array}{l}\text { Do not know } \\
\text { Yes } \\
\text { No }\end{array}$ & $\begin{array}{l}0.209 \\
0.547 \\
\text { Reference }\end{array}$ & $\begin{array}{l}1.232 \\
1.727\end{array}$ & $\begin{array}{l}0.827 \\
1.196\end{array}$ & $\begin{array}{l}1.834 \\
2.494\end{array}$ \\
\hline Sleep disturbance & $\begin{array}{l}\text { Yes } \\
\text { No }\end{array}$ & $\begin{array}{l}0.792 \\
\text { Reference }\end{array}$ & 2.208 & 1.601 & 3.044 \\
\hline Alcohol/SLT/others & $\begin{array}{l}\text { Yes } \\
\text { No }\end{array}$ & $\begin{array}{l}0.59 \\
\text { Reference }\end{array}$ & 1.804 & 1.319 & 2.466 \\
\hline
\end{tabular}

Abbreviations: AOR, adjusted odds ratio; $\mathrm{UCl}$, upper confidence interval; $\mathrm{LCl}$, lower confidence interval.

previous studies conducted by $\mathrm{CDC},{ }^{15}$ a study conducted in Bangladesh, ${ }^{38}$ and China. ${ }^{39-42}$ According to a previously conducted study, the low amount of physical activity increases the risk of severe COVID-19 infection. ${ }^{19}$ Our study did not observe any such association among Bangladeshi samples, which might be due to our study limitation (missing validated physical activity questionnaire). A more comprehensive study with validated questionnaire might be conducted to analyze such association.
Throughout the paper, we have discussed several risk factors of COVID-19 severe infection, and most of them are well known. Surprisingly, our analysis has suggested sleep disturbance as a significant predictor for life-threatening COVID-19. According to the previously conducted studies, sleep disturbance could increase the risk of CVD, hypertension, stroke, and various mental health issues. ${ }^{43}$ Moreover, the study has identified a higher risk COVID-19 among individuals with of greater sleep problems. ${ }^{18}$ 
Therefore, general people should give more attention to sound sleep. Our multiple logistic regression model indicates that there was no statistically significant association of COVID-19 severity with gender, occasional smoking, COPD, etc. According to a previous study, males were more likely to have severe infections, require ICU and had higher mortality rates than females. ${ }^{44}$ A study conducted in Korea identified COPD as a risk factor for increased severity, which supports the findings of our chi-square analysis. $^{42}$

\section{Strengths and Limitations}

Our study comes up with several strengths and limitations. We have successfully revealed some important risk factors of life-threatening COVID-19, which might be helpful for the general population as well as policymakers. On the other hand, the interview of the asymptomatic, mild, and moderate groups was conducted over a telephone conversation, which might be less efficient than a face-to-face interview. The convenient approach of sample selection was another limitation due to the shortage of manpower and time during the COVID-19 pandemic. We did not use validated questionnaires to measure physical activity level and sleep disturbance to shorten the interview. Moreover, our study did not address the dietary habits of the patients, though lifestyle is a combination of factors including diet, sedentary lifestyle, physical activity and sleep quality. A more comprehensive study covering all of Bangladesh's administrative divisions by following a more reliable sampling approach is highly recommended to get a more compact scenario.

\section{Conclusion}

This study has successfully revealed the lifestyle and comorbidity-related risk factors of severe-critical COVID-19 in Bangladesh. People with comorbidities should be under special consideration, and public health professionals should be aware of comorbidities and advance protection against COVID-19. With the help of mass media, the government's responsible authority should take awareness-raising programs and educate people on a healthy lifestyle (ie physical activity, regular breakfast, drink adequate water, avoids smoking and substance abuse). For a more fruitful control of severe-critical COVID-19 infection, public health professionals should pay immediate attention to the proper management of these risk factors and come up with appropriate action.

\section{Acknowledgments}

We would like to convey our heartfelt appreciation to the participants. We are also thankful to the hospital authorities for their support during data collection.

\section{Disclosure}

The authors report no conflicts of interest in this work.

\section{References}

1. Burrell CJ, Howard CR, Murphy FA. Epidemiology of viral infections. Fenner Whites Med Virol. 2017;185-203. doi:10.1016/B978-012-375156-0.00013-8

2. Cascella M, Rajnik M, Cuomo A, Dulebohn SC, Di Napoli R. Features, evaluation and treatment Coronavirus (COVID 19). StatPearls [Internet]; 2020. Available from: http://www.ncbi.nlm. nih.gov/pubmed/32150360. Accessed September 23, 2021.

3. Ghebreyesus TA. WHO director-general's opening remarks at the media briefing on COVID 19-11 March 2020 [Internet]. WHO Director General's speeches; 2020:4. Available from: https://www. who.int/director-general/speeches/who-director-general-s-openingremarks-at-the-media-briefing-on-COVID19-11-march-2020\% 0Ahttps://www.who.int/director-general/speeches/detail/who-direc tor-general-s-opening-remarks-at-the-media-briefing-on-cov.

Accessed September 23, 2021.

4. Johns Hopkins Coronavirus Resource Center. No title [Internet]; 2021 [cited July 13, 2021]. Available from: https://coronavirus.jhu. edu/map.html. Accessed September 23, 2021.

5. World Health Organization. Tracking SARS-CoV-2 variants [Internet]. Available from: https://www.who.int/en/activities/track ing-SARS-CoV-2-variants/. Accessed September 23, 2021.

6. Williamson EJ, Walker AJ, Bhaskaran K, et al. Factors associated with COVID 19-related death using OpenSAFELY. Nature. 2020;584 (7821):430-436. doi:10.1038/s41586-020-2521-4

7. Simonnet A, Chetboun M, Poissy J, et al. High prevalence of obesity in Severe Acute Respiratory Syndrome Coronavirus-2 (SARS-CoV2) requiring invasive mechanical ventilation. Obesity. 2020;28 (7):1195-1199. doi:10.1002/oby.22831

8. Centre for Disease Control and Prevention (CDC). COVID 19 [Internet]. Available from: https://www.cdc.gov/coronavirus/2019ncov/need-extra-precautions/index.html. Accessed September 23, 2021.

9. Schlesinger S, Neuenschwander M, Ballon A, Nöthlings U, Barbaresko J. Adherence to healthy lifestyles and incidence of diabetes and mortality among individuals with diabetes: a systematic review and meta-analysis of prospective studies. $J$ Epidemiol Community Health. 2020;74(5):481-487. doi:10.1136/jech-2019213415

10. Nyberg ST, Singh-Manoux A, Pentti J, et al. Association of healthy lifestyle with years lived without major chronic diseases. JAMA Intern Med. 2020;180(5):760-768. doi:10.1001/jamainternmed.20 20.0618

11. Li Y, Schoufour J, Wang DD, et al. Healthy lifestyle and life expectancy free of cancer, cardiovascular disease, and type 2 diabetes: prospective cohort study. BMJ. 2020;368:16669.

12. Simons D, Shahab L, Brown J, Perski O. The association of smoking status with SARS-CoV-2 infection, hospitalisation and mortality from COVID 19: a living rapid evidence review (version 6). Qeios. 2020;116:1319-1368.

13. World no tobacco day. Med Today. 2002;3:7. WHO: https://www. who.int/campaigns/world-no-tobacco-day

14. World Health Organization. WHO Statement: Tobacco Use and COVID 19. WHO; May, 2020:19-20. 
15. Centre for Disease Control and Prevention (CDC). People at Increased Risk of COVID 19; 2020:2019-2021.

16. Igić R. Smoking and COVID 19. Vojnosanit Pregl. 2020;77:461-462. doi:10.2298/VSP2005461I

17. Pötschke-Langer M, Schotte K, Szilagyi T. The WHO framework convention on tobacco control. Prog Respir Res. 2015;42:149-157.

18. Kim H, Hegde S, LaFiura C, et al. COVID-19 illness in relation to sleep and burnout. BMJ Nutr Prev Health. 2021;4(1):132-139. doi:10.1136/bmjnph-2021-000228

19. Hamer M, Kivimäki M, Gale CR, Batty GD. Lifestyle risk factors, inflammatory mechanisms, and COVID 19 hospitalization: a community-based cohort study of 387,109 adults in UK. Brain Behav Immun. 2020;87:184-187. doi:10.1016/j.bbi.2020.05.059

20. International Centre for Diarrhoeal Disease Research Bangladesh. Non-communicable diseases. https://www.icddrb.org/research/ research-themes/non-communicable-diseases

21. Nargis N, Thompson ME, Fong GT, et al. Prevalence and patterns of tobacco use in Bangladesh from 2009 to 2012: evidence from International Tobacco Control (ITC) study. PLoS One. 2015;10 (11):1-16. doi:10.1371/journal.pone.0141135

22. Fischer F, Minnwegen M, Kaneider U, Kraemer A, Khan MMH. Prevalence and determinants of secondhand smoke exposure among women in Bangladesh, 2011. Nicotine Tob Res. 2015;17(1):58-65. doi:10.1093/ntr/ntu129

23. Coronavirus disease (COVID-2019) Bangladesh situation reports. https://www.who.int/bangladesh/emergencies/coronavirus-disease(covid-19)-update/coronavirus-disease-(covid-2019)-bangladeshsituation-reports

24. Bangladesh Society of Medicine. 2019-nCOV (SARS-COV-2) Infection Prevention Control (IPC) and Management in Healthcare Facilities; 2020.

25. Ho FK, Petermann-Rocha F, Gray SR, et al. Is older age associated with COVID 19 mortality in the absence of other risk factors? General population cohort study of 470,034 participants. PLoS One. 2020;15(11):e0241824. doi:10.1371/journal.pone.0241824

26. Boehmer TK, DeVies J, Caruso E, et al. Changing age distribution of the COVID 19 pandemic. MMWR Morb Mortal Wkly Rep. 2020;69 (39):1404-1409. doi:10.15585/mmwr.mm6939e1

27. Cortis D. On determining the age distribution of COVID 19 pandemic. Front Public Health. 2020;8(May):1-3.

28. Harlem G. Descriptive analysis of social determinant factors in urban communities affected by COVID 19. J Public Health. 2020;42 (3):466-469.

29. Demeulemeester F, de Punder K, van Heijningen M, van Doesburg F. Obesity as a risk factor for severe COVID 19 and complications: a review. Cells. 2021;10(4). doi:10.3390/cells10040933

30. Gao F, Zheng KI, Wang XB, et al. Obesity is a risk factor for greater COVID 19 severity. Diabetes Care. 2020;43(7):E72-E74. doi:10.23 $37 / \mathrm{dc} 20-0682$
31. World Health Organization. Update: 31 July 2020. WHO; July, 2020:1-203.

32. Berlin I, Thomas D, Le Faou AL, Cornuz J. COVID 19 and smoking. Nicotine Tob Res. 2020;22(9):1650-1652. doi:10.1093/ntr/ntaa059

33. Sanyaolu A, Okorie C, Marinkovic A, et al. Comorbidity and its impact on patients with COVID 19. SN Compr Clin Med. 2020;2 (8):1069-1076. doi:10.1007/s42399-020-00363-4

34. Zhou Y, Yang Q, Chi J, et al. Comorbidities and the risk of severe or fatal outcomes associated with coronavirus disease 2019: a systematic review and meta-analysis. Int $J$ Infect Dis. 2020;99:47-56. doi:10.1016/j.ijid.2020.07.029

35. Wu J, Zhang J, Sun X, et al. Influence of diabetes mellitus on the severity and fatality of SARS-CoV-2 (COVID 19) infection. Diabetes Obes Metab. 2020;22(10):1907-1914. doi:10.1111/dom.14105

36. Nishiga M, Wang DW, Han Y, Lewis DB, Wu JC. COVID 19 and cardiovascular disease: from basic mechanisms to clinical perspectives. Nat Rev Cardiol. 2020;17(9):543-558. doi:10.1038/s41569020-0413-9

37. Ortiz A, Cozzolino M, Duivenvoorden R, et al. Chronic kidney disease is a key risk factor for severe COVID 19: a call to action by the ERA-EDTA. Nephrol Dial Transplant. 2021;36(1):87-94. doi:10.1093/ndt/gfaa314

38. Mohsin F, Tonmon T, Nahrin R, et al. Association between smoking and COVID 19 severity: evidence from Bangladesh. $J$ Multidiscip Healthc. 2021;14:1923-1933. doi:10.2147/JMDH.S317603

39. Zhang JJ, Dong X, Cao YY, et al. Clinical characteristics of 140 patients infected with SARS-CoV-2 in Wuhan, China. Allergy Eur $J$ Allergy Clin Immunol. 2020;75(7):1730-1741. doi:10.1111/all.14238

40. Zhou F, Yu T, Du R, et al. Clinical course and risk factors for mortality of adult inpatients with COVID 19 in Wuhan, China: a retrospective cohort study. Lancet. 2020;395(10229):1054-1062. doi:10.1016/S0140-6736(20)30566-3

41. Cen Y, Chen X, Shen Y, et al. Risk factors for disease progression in patients with mild to moderate coronavirus disease 2019-a multicentre observational study. Clin Microbiol Infect. 2020;26(9):12421247. doi:10.1016/j.cmi.2020.05.041

42. Zhao Q, Meng M, Kumar R, et al. The impact of COPD and smoking history on the severity of COVID 19: a systemic review and metaanalysis. $J$ Med Virol. 2020;92(10):1915-1921. doi:10.1002/ jmv. 25889

43. Newman AB, Enright PL, Manolio TA, Haponik EF, Wahl PW. Sleep disturbance, psychosocial correlates, and cardiovascular disease in 5201 older adults: the Cardiovascular Health Study. J Am Geriatr Soc. 1997;45(1):1-7. doi:10.1111/j.1532-5415.1997.tb00970.x

44. Vahidy FS, Pan AP, Ahnstedt H, et al. Sex differences in susceptibility, severity, and outcomes of coronavirus disease 2019: crosssectional analysis from a diverse US metropolitan area. PLoS One. 2021;16(1):e0245556. doi:10.1371/journal.pone.0245556
Infection and Drug Resistance

\section{Publish your work in this journal}

Infection and Drug Resistance is an international, peer-reviewed openaccess journal that focuses on the optimal treatment of infection (bacterial, fungal and viral) and the development and institution of preventive strategies to minimize the development and spread of resistance. The journal is specifically concerned with the epidemiology of antibiotic resistance and the mechanisms of resistance development and diffusion in both hospitals and the community. The manuscript management system is completely online and includes a very quick and fair peerreview system, which is all easy to use. Visit http://www.dovepress.com/ testimonials.php to read real quotes from published authors. 